\title{
PERSONALIDAD DE MARCA DE CARRERAS DE PSICOLOGÍA DE UNIVERSIDADES ESTATALES EN CHILE: UN ESTUDIO DESCRIPTIVO
}

\author{
Marianela Denegri C. ${ }^{1}$, Daniel Cabezas G., Viviana Herrera C., Alex Páez S., Mauricio Vargas Z. \\ Universidad de La Frontera, Chile \\ (RECBBIDO EL 3/11/2009, ACEPTADO EL 2/12/ 2009)
}

\begin{abstract}
RESUMEN
El objetivo de la presente investigación, que forma parte de un macro estudio con el consorcio de Escuelas de Psicología de universidades estatales chilenas, fue determinar la existencia de una personalidad de marca para las carreras de Psicología de dos universidades estatales integrantes de este consorcio. Los participantes fueron 289 estudiantes de la carrera de psicología de la Universidad de Santiago de Chile (Región Metropolitana) y de la Universidad de La Frontera (Región de La Araucanía). Para esto, se aplicó a los participantes la Escala de Personalidad de Marca de Aaker, la cual se compone de 45 ítems donde cada uno representa un rasgo de la personalidad de marca en estudio. Los resultados muestran la existencia de una Personalidad de Marca definida para cada carrera, con diferencias en los rasgos constitutivos según la universidad. Se discute las implicancias de contar con una personalidad de marca con rasgos definidos, tanto en calidad como en cantidad, en un mercado universitario competitivo en el que la diferenciación y el aseguramiento de la calidad son desafíos de toda institución de educación superior en la actualidad.
\end{abstract}

Palabras clave: Personalidad de marca, universidades estatales, mercado universitario.

\section{ABSTRACT \\ BRAND PERSONALITY IN PSYCHOLOGY CAREER OF STATE UNIVERSITIES IN CHILE: A DESCRIPTIVE STUDY}

The following research attempts to determinate the existence of a Brand Personality among two Chilean State Universities' Psychology undergraduate programs. The Aaker's Brand Personality Scale was applied on 289 students of both undergraduate programs in order to evaluate their impressions for 45 characteristics that are included in the scale. Results show the existence of a defined Brand Personality for each program, with one of them includes different characteristics. The advantage of a defined Brand Personality in a complex scenario for universities is discussed, including a reflection about the importance of stating differences on high education services.

Keywords: Brand personality, state universities, high education market.

1 Docente investigadora del Centro de Excelencia en Psicología Económica y del Consumo, Universidad de La Frontera, Chile. E-mail: mdenegri@gmail.com 


\section{INTRODUCCIÓN}

El rápido avance de los procesos de globalización en Chile en las últimas décadas ha llevado a que el Sistema de Educación Superior expanda y diversifique su oferta, expresándose específicamente en una masificación y pronta saturación de profesionales del área de la Psicología en el país. El diagnóstico de la situación actual de la profesión (Consejo Superior de Educación, 2005) muestra un proceso creciente de aumento de la oferta de vacantes en el sistema privado, gran diferenciación de las instituciones formadoras y de sus múltiples 'sedes' lo que ha implicado un aumento vertiginoso de la cantidad de programas y escuelas de Psicología, principalmente durante la última década (Brunner, 2007).

En este escenario de creciente competitividad y oferta ante una demanda que no crece a la misma velocidad, cada escuela de Psicología se enfrenta a la necesidad de diferenciarse entre las múltiples instituciones que imparten esta carrera, a fin de sostenerse en el tiempo y lograr desarrollar su proyecto educativo de forma óptima. Con el objetivo de conocer las impresiones que los usuarios y miembros de cada organización poseen sobre la misma, así como para la articulación de estrategias de difusión y posicionamiento más eficientes, la identificación de la Personalidad de Marca de una escuela de Psicología constituye un significativo aporte que permitiría la construcción de una imagen corporativa que revista sentido para los usuarios-clientes.

Según la Asociación Americana de Marketing ( $A M A)$, una marca es un nombre, término, signo, diseño o una combinación de ellos, pensado para identificar al producto o servicio y para diferenciarlos de la competencia (Mercado, 2004). En función de lo anterior, la imagen de marca se puede definir como el conjunto de percepciones, asociaciones, recuerdos y prejuicios que el público procesa en su cabeza y cuya síntesis es una imagen mental del producto y de las ventajas y beneficios que los consumidores reciben o creen que pueden recibir a través de su uso (Mercado, 2004)

Las marcas cumplen así los roles fundamentales de identificar, significar y diferenciar los distintos productos o servicios que se ofrecen en el mercado, de modo que éstos logren notoriedad con respecto a sus competidores mediante la formación de asociaciones de significado vinculadas al producto que conforman finalmente la imagen simbólica en el imaginario del usuario-consumidor (Olavarrieta, 2002; Boubeta, Varela, Braña Tobio y Mangin, 2000; Plummer, 1985; Ramaseshan, Hsiu-Yuan, 2007; Park, Jaworski y Maclnnis, 1986; Keller, 1993).

Diversos autores señalan (Boubeta et al., 2000; Ramaseshan, Hsiu-Yuan, 2007) que vinculada a la imagen de marca, el consumidor identifica algún tipo de beneficio que ésta pueda otorgarle, los que pueden ser funcionales, es decir, aquel que le permita resolver determinados problemas; simbólicos, vinculados a la imagen de sí mismos o la pertenencia a algún grupo de referencia; o bien experienciales, aludiendo a aquellas marcas que les permitan obtener sensaciones placenteras o estímulos cognitivos. Esto permite que los individuos que seleccionan una marca en particular desarrollen un vínculo afectivo, de lealtad y compromiso con ella, siendo esto uno de los mayores predictores del éxito de dicha marca en el mercado (Boubeta et al., 2000). 
Plumer (1985) añade que existen tres componentes fundamentales en la imagen de una marca que permiten la descripción de la misma, éstas son; aspecto físico o atributos; las características funcionales, esto es, las consecuencias derivadas de su uso y por último, las características de la marca o su personalidad. Estos elementos interactúan de tal manera que los consumidores consideran que determinada marca es adecuada para ellos.

La idea de que características intangibles de las marcas constituyen una fuente de riqueza, ha determinado que actividades de creación, desarrollo y adquisición de marcas ya establecidas sean consideradas como alternativas de inversión (Delgado, 2004). El concepto de personalidad de marca generó un cambio en la forma de entender como los consumidores establecen sus preferencias hacia una marca (Aaker, 1996); contrastando con el concepto de "atributos del producto" (visto como una función meramente utilitaria). La marca y la personalidad prestan una función simbólica o de autoexpresión para el individuo, convirtiéndose en la manera en la que perciben a los demás y a sí mismos (Keller, 1993). Los consumidores se asocian emocionalmente con sus marcas y piensan en ellas como personas, atribuyéndoles características humanas y rasgos de personalidad, bien sea por similitud ("esta marca se parece a mí") o posesión ("esta es mi marca"). Al definir personalidad de marca como el "set de características humanas asociadas a la marca" (Aaker, 1997:347), se establece un vínculo entre los rasgos de personalidad de los individuos y los atribuidos a la marca, las cuales sirven a los consumidores como un medio simbólico para expresarse a sí mismos (Ligas, 2000; Fournier, 1991), representando los valores y las creencias culturales de un grupo en específico (Sung y Tinkham, 2005; Aaker, 1992; Keller, 1993; Belch y Belch, 1998; Tybout y Carpenter, 2002).

A pesar de que existen una serie de métodos para medir la personalidad de marca, uno de los más difundidos es el desarrollado por Aaker (1997) a partir de una serie de estudios empíricos realizados en Estados Unidos. Desde su publicación, el modelo de Aaker (1997) ha sido replicado y adaptado en diversos países en todo el mundo, tales como Francia (Koebel y Ladwein, 1999), Japón, España (Aaker, Benet-Martínez y Garo-2001), México (Álvarez-Ortiz y Harris, 2002), Rusia (Supphellen y Grohaug, 2003) y Venezuela, (Barrios y Massa, 1999 y Pirela, Villavicencio y Saavedra, 2004).

El modelo mencionado consta de cinco dimensiones principales de personalidad en las marcas, cada una de las cuales está conformada por una serie de rasgos distintivos, y que para el presente estudio ha sido adaptado tal como se indica en la Figura N. ${ }^{0} 1$. 


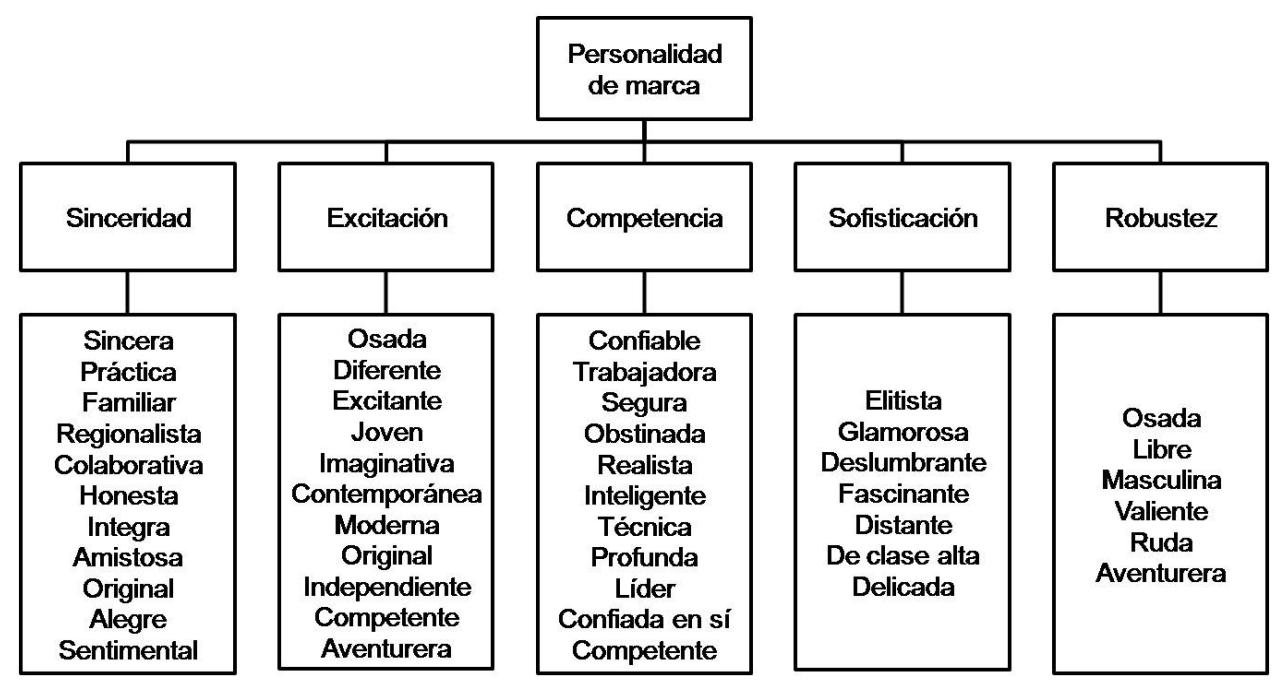

Figura N. ${ }^{0}$ 1. Componentes del modelo de personalidad de marca de Aaker (1997).

Sin embargo, existen muy pocos estudios en organizaciones orientadas al servicio como son las universidades. Los resultados de los estudios señalan la relación entre la percepción de personalidad de marca y la aceptación de la imagen corporativa así como relación significativa entre satisfacción con los servicios recibidos y rasgos positivos de personalidad de marca (Berli, Díaz y Pérez, 2002; Minjung Sung y; Sung-Un Yang, 2008).

De esta manera, se hace necesario identificar elementos que logren diferenciar de manera clara a estas carreras en el mercado universitario actual a la vez que potenciar aquellos elementos comunes que diferencian a la formación en psicología en la universidad estatal de aquellas que pertenecen al ámbito privado. Para ello es esencial conocer la percepción que tienen los usuarios (estudiantes) en relación con las características que distinguen a las instituciones en que se están formando y determinar con ello la valoración que otorgan a la imagen de cada carrera.

A partir de esto, el objetivo de la presente investigación fue determinar la personalidad de marca de dos carreras de psicología de universidades estatales de Chile: la Universidad de Santiago de Chile y la Universidad de La Frontera.

Al mismo tiempo se pretendía validar un instrumento que permitiera evaluar este constructo en servicios educacionales de nivel terciario.

\section{MÉTODO}

\section{Participantes}

La población de estudio la constituyeron todos los estudiantes de dos carreras de psicología de universidades estatales. El tipo de muestreo fue estratificado con afijación simple con un 
error Tipo I máximo de 0.05 y una potencia de prueba de 0.90 , quedando conformada la muestra por 283 estudiantes de Psicología, 138 de ellos correspondientes a la Universidad de Santiago de Chile (USACH) y 145 a la Universidad de La Frontera, los que se encontraban cursando entre el primer y el quinto año. La distribución por género fue de 184 mujeres $(65 \%)$ y 99 hombres (35\%), reflejando la estructura de género tradicional al interior de esta formación.

La participación fue voluntaria y todos los participantes firmaron un consentimiento informado donde accedían a participar en el estudio. Un documento similar fue firmado por las autoridades responsables de cada carrera involucrada.

\section{Instrumento}

El instrumento con el cual se recolectaron los datos es la "Escala de personalidad de marca de Aaker" (1997). Esta escala contiene 45 ítems, donde cada uno representa un rasgo de personalidad de marca en estudio. Cabe destacar que al instrumento original se han incluido tres rasgos ("Clase baja", "Clase media" y "Femenina"), los cuales resultan relevantes de acuerdo al contexto local de la investigación. El formato de respuesta es de tipo Likert, con una escala de 1 a 5 en función con el desacuerdo o acuerdo para cada ítem. Este instrumento cuenta con un índice de confiabilidad Alfa de Cronbach de 0,90, conteniendo la totalidad de los ítems iniciales.

\section{Procedimiento}

Previo a la realización del estudio se mantuvieron reuniones con las autoridades responsables de cada carrera involucrada, los que fueron informados de los objetivos y alcances de la investigación y a los cuales se solicitó su conformidad mediante la firma de un consentimiento informado.

El instrumento fue aplicado por el equipo de investigación en los cursos regulares de las carreras mencionadas, donde se les explicó a los estudiantes el propósito del estudio y se solicitó su consentimiento informado para participar, asegurando además la confidencialidad de sus respuestas. La modalidad de aplicación fue autoadministrada, con un tiempo de aplicación aproximado de 15 minutos.

\section{Plan de análisis}

Los datos recolectados se analizaron a través del paquete estadístico SPSS v.15. Se utilizaron estadísticos descriptivos para conocer las medias de las 45 variables, Pruebas de confiabilidad y Pruebas de muestras independientes para comparar a ambas instituciones con relación a los factores y las variables que conforman la personalidad de marca percibida entre los distintos niveles del establecimiento, así como para realizar comparaciones de género en cada carrera. 


\section{RESULTADOS}

Al observar las medias de las 45 variables de la Escala de Personalidad de Marca, se encontró que para la carrera de Psicología de la Universidad de Santiago de Chile, 15 de ellas se encuentran en el rango más alto (sobre 3,5), por lo que son percibidas como distintivas de la carrera, entre los que destacan rasgos como Competente $(3,99)$, Inteligente $(3,86)$, Trabajadora $(3,84)$, Amistosa $(3,82)$ y Realista $(3,80)$. (ver Tabla N. $\left.{ }^{\circ} 1\right)$.

Tabla N. ${ }^{\circ}$ 1. Rasgos característicos (Universidad de Santiago de Chile).

\begin{tabular}{lcc}
\hline Rasgos & Media & Desviación típica \\
\hline Práctica & 3,64 & 0,862 \\
Colaborativa & 3,71 & 0,766 \\
Íntegra & 3,73 & 0,900 \\
Amistosa & 3,82 & 0,882 \\
Alegre & 3,59 & 0,918 \\
Joven & 3,58 & 1,017 \\
Contemporánea & 3,54 & 0,897 \\
Competente & 3,99 & 0,888 \\
Confiable & 3,70 & 0,825 \\
Trabajadora & 3,84 & 0,938 \\
Segura & 3,54 & 0,864 \\
Realista & 3,80 & 0,794 \\
Inteligente & 3,86 & 0,803 \\
Confiada & 3,70 & 0,866 \\
Clase media & 3,71 & 1,166 \\
\hline
\end{tabular}

Ahora, para la carrera de Psicología de la Universidad de La Frontera, 18 de los 45 rasgos se encuentran en el rango más alto, destacándose: Competente $(4,01)$, Amistosa $(3,94)$, Confiable $(3,90)$, Inteligente $(3,86)$ y Profunda $(3,86)$ (ver Tabla N. $\left.{ }^{\circ} 2\right)$.

Tabla N. ${ }^{\circ}$ 2. Rasgos característicos (Universidad de La Frontera).

\begin{tabular}{lcc}
\hline Rasgos & Media & Desviación típica \\
\hline Práctica & 3,62 & 0,867 \\
Familiar & 3,74 & 0,993 \\
Colaborativa & 3,72 & 0,917 \\
Honesta & 3,63 & 0,920 \\
Íntegra & 3,79 & 1,001 \\
Amistosa & 3,94 & 0,907 \\
Alegre & 3,61 & 0,959 \\
Diferente & 3,67 & 0,951 \\
Imaginativa & 3,57 & 1,026 \\
Contemporánea & 3,67 & 0,972 \\
Competente & 4,01 & 0,909 \\
Confiable & 3,90 & 0,892 \\
Trabajadora & 3,63 & 0,881 \\
Segura & 3,66 & 0,860 \\
Realista & 3,79 & 0,781 \\
Inteligente & 3,86 & 0,925 \\
Profunda & 3,86 & 0,962 \\
Confiada & 3,67 & 0,921 \\
\hline
\end{tabular}


$\mathrm{Al}$ analizar las variables agrupadas en los cinco factores planteados por Aaker de acuerdo a la universidad que imparte la carrera de Psicología, se encontró que en el caso de la Universidad de Santiago de Chile sólo el factor Competencia $(3,52)$ sería característico de ella. Los factores que siguen en puntaje, pero que no serían característicos son: Sinceridad $(3,36)$ y Excitación $(3,29)$.

Para el caso de la carrera de Psicología de la Universidad de La Frontera, los factores que puntúan más alto son Competencia $(3,55)$, Sinceridad $(3,54)$ y Excitación $(3,30)$, de los cuales serían característicos de la carrera los factores Competencia y Sinceridad. (Ver Tabla N. ${ }^{\circ}$ 3).

Tabla N. ${ }^{\circ}$ 3. Medias factores por universidad.

\begin{tabular}{llcccc}
\hline \multirow{2}{*}{ Universidad } & N & Media & Desviación típ. & $\begin{array}{c}\text { Error típ. de } \\
\text { la media }\end{array}$ \\
\hline \multirow{2}{*}{ Sinceridad } & Univ. de La Frontera & 145 & 3,5448 & 0,60317 & 0,05009 \\
& Univ. de Santiago de Chile & 138 & 3,3682 & 0,49517 & 0,04215 \\
\multirow{2}{*}{ Excitación } & Univ. de La Frontera & 145 & 3,3066 & 0,67001 & 0,05564 \\
& Univ. de Santiago de Chile & 138 & 3,2918 & 0,61181 & 0,05208 \\
\multirow{2}{*}{ Competencia } & Univ. de La Frontera & 145 & 3,5530 & 0,53338 & 0,04429 \\
& Univ. de Santiago de Chile & 138 & 3,5217 & 0,53589 & 0,04562 \\
\multirow{2}{*}{ Sofisticación } & Univ. de La Frontera & 145 & 2,4749 & 0,57977 & 0,04815 \\
& Univ. de Santiago de Chile & 138 & 2,2619 & 0,51597 & 0,04392 \\
\multirow{2}{*}{ Robustez } & Univ. de La Frontera & 145 & 2,5805 & 0,58469 & 0,04856 \\
& Univ. de Santiago de Chile & 138 & 2,7560 & 0,57530 & 0,04897 \\
\hline
\end{tabular}

Al realizar una comparación de los factores de acuerdo a la variable género en cada una de las carreras, se observa que no existirían diferencias significativas en el caso de la Universidad de Santiago de Chile, observándose una diferencia de medias más elevada en el caso de los factores Competencia (3,57 las mujeres y 3,40 los hombres) y en el factor Excitación (3,35 las mujeres y 3,15 los hombres). (Ver Tabla N. ${ }^{\circ}$ ).

Tabla N. ${ }^{\circ}$. Factores por sexo (Universidad de Santiago de Chile).

\begin{tabular}{|c|c|c|c|c|c|}
\hline & Sexo & $\mathbf{N}$ & Media & Desviación típ. & Error típ. de la media \\
\hline \multirow{2}{*}{ Sinceridad } & Mujer & 93 & 3,3812 & ,46166 & ,04787 \\
\hline & Hombre & 45 & 3,3414 &, 56279 & 08390 \\
\hline \multirow{2}{*}{ Excitación } & Mujer & 93 & 3,3568 &, 57827 & ,05996 \\
\hline & Hombre & 45 & 3,1576 & ,66251 & ,09876 \\
\hline \multirow{2}{*}{ Competencia } & Mujer & 93 & 3,5787 & ,49861 &, 05170 \\
\hline & Hombre & 45 & 3,4040 &, 59441 &, 08861 \\
\hline \multirow{2}{*}{ Sofisticación } & Mujer & 93 & 2,2028 & ,54166 &, 05617 \\
\hline & Hombre & 45 & 2,3841 & ,43907 &, 06545 \\
\hline \multirow{2}{*}{ Robustez } & Mujer & 93 & 2,7437 & ,56975 & 05908 \\
\hline & Hombre & 45 & 2,7815 & ,59227 &, 08829 \\
\hline
\end{tabular}


En el caso del análisis por sexo en la carrera de Psicología de la Universidad de La Frontera, se observa que no existiría diferencias significativas en ninguno de los factores de la personalidad de marca, pero sí se observan diferencias de medias, especialmente en los factores Competencia (3,60 las mujeres y 3,46 los hombres) y Sinceridad (3,61 las mujeres y 3,42 los hombres) (ver Tabla N. ${ }^{\circ}$ ).

Tabla N. ${ }^{0}$ 5. Factores por sexo (Universidad de La Frontera).

\begin{tabular}{|c|c|c|c|c|c|}
\hline & Sexo & $\mathbf{N}$ & Media & Desviación típ. & Error típ. de la media \\
\hline \multirow{2}{*}{ Sinceridad } & Mujer & 91 & 3,6134 & 0,53295 & 0,05587 \\
\hline & Hombre & 54 & 3,4293 & 0,69603 & 0,09472 \\
\hline \multirow{2}{*}{ Excitación } & Mujer & 91 & 3,3506 & 0,64703 & 0,06783 \\
\hline & Hombre & 54 & 3,2323 & 0,70698 & 0,09621 \\
\hline \multirow{2}{*}{ Competencia } & Mujer & 91 & 3,6024 & 0,51935 & 0,05444 \\
\hline & Hombre & 54 & 3,4697 & 0,55107 & 0,07499 \\
\hline \multirow{2}{*}{ Sofisticación } & Mujer & 91 & 2,4490 & 0,54234 & 0,05685 \\
\hline & Hombre & 54 & 2,5185 & 0,64086 & 0,08721 \\
\hline \multirow{2}{*}{ Robustez } & Mujer & 91 & 2,5604 & 0,56629 & 0,05936 \\
\hline & Hombre & 54 & 2,6142 & 0,61841 & 0,08415 \\
\hline
\end{tabular}

\section{DISCUSIÓN}

Los resultados obtenidos en los análisis descriptivos generales para cada carrera de Psicología permiten observar cómo en el caso de la carrera de Psicología de la Universidad de Santiago de Chile, se aprecian una serie de rasgos claramente agrupados en los factores competencia y sinceridad, lo cual denota una personalidad de marca relativamente clara y explícita, que se proyecta hacia los usuarios de la carrera a través de atributos identificables y de manera transversal, sin diferencias según el género de los estudiantes. Además, destacan dos factores que la diferencian de la misma carrera en la Universidad de La Frontera, lo que podría estar relacionado con los rasgos joven y clase media, ya que esta carrera tiene un antigüedad de quince años, así como por el hecho de ser una universidad estatal que recibe a estudiantes de un segmento económico determinado y que corresponde a la mayoría de la población del país.

Por otra parte, los resultados muestran que la carrera de Psicología de la Universidad de La Frontera presenta una cantidad relativamente mayor de rasgos distintivos en comparación con la misma carrera de la Universidad de Santiago de Chile, donde destacan características ligadas a los factores competencia, sinceridad y excitación, y que permiten apreciar una personalidad de marca definida.

Adicionalmente, es posible destacar que tanto en la carrera de Psicología de la Universidad de La Frontera como en la de la Universidad de Santiago de Chile, hombres como mujeres tienen una visión similar de los factores característicos de la carrera, deducción que se deriva 
de la ausencia de diferencias estadísticamente significativas en ambos casos. Este resultado permitiría descartar la existencia de un sesgo en función del género de los estudiantes, lo cual se diferencia de los hallazgos de Denegri, Etchebarne, Geldres, Cabezas y Herrera (2009) en la carrera de Ingeniería Comercial de la Universidad de La Frontera. De esta forma, es posible distinguir claras diferencias en las trayectorias de ambas carreras a pesar de ser parte del mismo plantel.

Así, el hecho de que ambas carreras presenten una personalidad de marca definida implica que cada una de ellas estaría siendo coherente tanto en sus acciones como en su discurso, lo cual favorecería su posicionamiento, en virtud de lo señalado por Kapferer (2003) en relación con que una marca gana personalidad de acuerdo con su discurso y comportamiento; así adquiere un carácter, al colocar, de esta forma, en evidencia el tipo de persona que ella tendría.

De este modo, se puede sintetizar que la marca se consolida como un elemento de valor para los consumidores y usuarios, ya que ésta asegura un determinado nivel de calidad, simplifica un proceso de elección y, especialmente, se constituye en una motivación para que los individuos consigan una gran cantidad de metas en su vida privada y pública, determinando desde la satisfacción de necesidades fisiológicas básicas, hasta autorrealización personal (Aaker, 1992; Keller, 1993; Belch y Belch, 1998; Stanton, Etzel y Walker, 2000; Tybout y Carpenter, 2002).

\section{CONCLUSIONES}

Los principales hallazgos de esta investigación establecen la existencia de una personalidad de marca para cada una de las carreras participantes, estando presente en ambos casos el factor competencia. Esta constatación resulta relevante ya que la opción por una determinada casa de estudios implica una decisión trascendental en la formación profesional y en el desarrollo laboral posterior de los estudiantes, por lo cual la percepción de que con la alternativa seleccionada obtendrán un adecuado nivel de capacidades les permite tener seguridad acerca de la elección realizada.

Lo anterior se relaciona directamente con el alto grado de competitividad que ha alcanzado el mercado laboral derivado de la amplia oferta en la formación de pregrado en psicología (Brunner, 2007), lo cual requiere que los estudiantes y egresados sientan confianza tanto en las competencias adquiridas, así como también en cuanto al prestigio del plantel universitario en el cual cursaron sus estudios.

En este sentido, resulta significativo que ambas carreras posean rasgos distintivos y que éstos se vinculen con características como Inteligencia, Confianza en sí y Seguridad, ya que en algún nivel, estas apreciaciones se relacionan con beneficios que el usuario-cliente demanda y requiere según (Boubeta et al., 2000; Ramaseshan, Hsiu-Yuan, 2007) y que, en el contexto actual, resultan altamente significativos en términos de la futura empleabilidad de los estudiantes.

Finalmente, los resultados entregados son de gran importancia para el diseño de estrategias de posicionamiento y difusión del trabajo realizado en ambas escuelas de psicología, ya 
que tanto la Universidad de Santiago de Chile y la Universidad de La Frontera pertenecen al Consorcio de Universidades Estatales, las cuales se enfrentan a un sistema de educación superior de alta complejidad, donde la participación privada alcanza, en términos generales, el 74\% del total de la matrícula (CINDA, 2007), lo cual supone el permanente desafío de la competencia por alumnos, recursos y reputación (Brunner 2006), y que para el caso de las carreras de psicología, no superan el 2,6\% de la matrícula total del área.

\section{REFERENCIAS BIBLIOGRÁFICAS}

1. Aaker, D. (1992). The value of brand equity. Journal of Business Strategy. 13(4), 27-32.

2. Aaker, J. (1997). Dimensions of Brand Personality. Journal of Marketing Research. Vol. 34, 347-356.

3. Aaker, J.; Benet-Martinez, V. y Gorolera, J. (2001). Consumption symbols as carriers of culture: A study of Japanese and Spanish brand personality constructs, Journal of Personality and Social Psychology, 81(3) 492-508.

4. Álvarez-Ortiz, C. y Harris, J. (2002). Assesing the structure of brand personality among global and local Mexican brands. 2002 AMA Summer Educators Conference, San Diego, Estados Unidos.

5. Barrios, M. y Massa, C. (1999). Dimensiones de la personalidad de marca en Venezuela. Tesis de Maestría, Instituto de Estudios Superiores de Administración.

6. Belch, G. y Belch, M. (1998). Advertising and Promotion. USA: Irwin/McGrawHill.

7. Berli, A.; Díaz, G.; Pérez, P. (2002). The configuration of the university image and its relationship with the satisfaction of students. Journal of Educational Administration, Vol. 40- 486-505

8. Boubeta, A.; Varela, J.; Braña Tobio, T. y Levy, J.P. (2000). El valor de la marca a partir de su relación con el consumidor. Psicothema, 12(2) 247-254.

9. Brunner, J. (2007). Mercados universitarios: los nuevos escenarios de la educación superior. Informe Final de Proyecto FONDECYT N. ${ }^{0} 1050138$.

10. Brunner, J. (2006). Sistema privatizado y mercados universitarios: competencia reputacional y sus efectos. Informe elaborado en el marco del proyecto FONDECYT N. ${ }^{\circ} 1050138$.

11. Centro Interuniversitario de Desarrollo (CINDA) (2007). La Educación Superior en Iberoamérica: Informe 2007. Santiago: RIL Editores.

12. Consejo Superior de Educación (2005) Índices. Chile.

13. Denegri, M.; Etchebarne, S.; Geldres, V.; Cabezas, D. y Herrera, V. (2009). Personalidad de marca de las carreras de ciencias empresariales: un análisis comparativo entre universidad pública y privada. Trabajo presentado en la XLIV 
Asamblea Anual del Consejo Latinoamericano de Escuelas de Administración (CLADEA), Noviembre, Guayaquil.

14. Fournier, S. (1991) A meaning-based framework for the study of consumer - object relations. In R. Holman and M. Soloman (Eds.) Advances in Consumer Research, 18, 736-42, Provo, UT: Association for Consumer Research.

15. Kapferer, Jean-Noël (2003). As marcas, capital da empresa: criar e desenvolver marcas fortes. Porto Alegre: Bookman.

16. Keller, K. (1993). Conceptualizing, measuring, and managing customer-based brand equity. Journal of Marketing. 57(1), 1-22.

17. Koebel, M. y Ladwein, R. (1999). L'échelle de personnalité de la marque de Jennifer Aaker: Adaptation au contexte français. Decisions Marketing, 18, 81-88.

18. Ligas, M. (2000) People, products and pursuits: exploring the relationship between consumer goals and product meanings, Psychology and Marketing, 17, 983-1003.

19. Mercado, V. (2004). Ranking general de marcas chilenas. Tesis para optar al título de Ingeniero Comercial, Universidad de Chile.

20. Minjung Sung a; Sung-Un Yang (2008) Toward the Model of University Image: The Influence of Brand Personality, External Prestige and Reputation. Journal of Public Relations Research, 20(4), 357-376.

21. Olavarrieta, S. (2002). ¿Por qué tiene valor su marca para los consumidores? Revista Economía y Administración, 143, 21-29.

22. Plummer, J. (1985). How does personality makes a difference. Journal of Advertising Research. 21, 27-31.

23. Pirela, J. L.; Villavicencio, H. and Saavedra, J. L. (2004). Dimensiones de personalidad de marca: estudio exploratorio de los rasgos de personalidad en Venezuela. Revista de Ciencias Sociales, 10 (3), 430-440.

24. Park, C.V.; Jaworski, B.J.; MacInnis, D.J. (1986). Strategic brand concept-image management. Journal of Marketing, 50, 135-145.

25. Ramaseshan, B.; Hsiu-Yuan, Tsao (2007). Moderating effects on the brand concept on the relationship between brand personality and perceived quality. Journal of Brand Management, 14, 458-466.

26. Stanton, W.; Etzel, M. y Walker, B. (2000). Fundamentos de marketing. México: McGraw-Hill Interamericana.

27. Suppehellen, M. y Gornhaug, K. (2003). Building foreign brand personalities in Russia: the moderating effect of consumer ethnocentrism. International Journal of Advertising, 22(2) 203-226.

28. Tybout, A. y Carpenter, G. (2002). Crear y administrar marcas. Marketing según Kellogg. España: Vergara Editores / Business. 